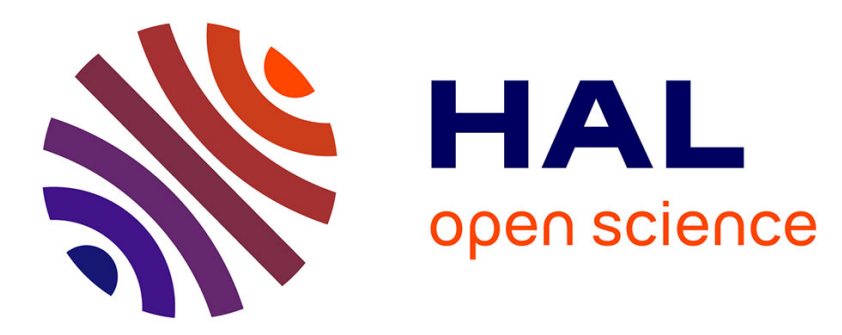

\title{
Subwavelength seismic metamaterial with an ultra-low frequency bandgap
}

Yi Zeng, Pai Peng, Qiu-Jiao Du, Yue-Sheng Wang, B. Assouar

\section{To cite this version:}

Yi Zeng, Pai Peng, Qiu-Jiao Du, Yue-Sheng Wang, B. Assouar. Subwavelength seismic metamaterial with an ultra-low frequency bandgap. Journal of Applied Physics, 2020, 128 (1), pp.014901. $10.1063 / 1.5144177$. hal-03043181

\section{HAL Id: hal-03043181 \\ https://hal.science/hal-03043181}

Submitted on 7 Dec 2020

HAL is a multi-disciplinary open access archive for the deposit and dissemination of scientific research documents, whether they are published or not. The documents may come from teaching and research institutions in France or abroad, or from public or private research centers.
L'archive ouverte pluridisciplinaire HAL, est destinée au dépôt et à la diffusion de documents scientifiques de niveau recherche, publiés ou non, émanant des établissements d'enseignement et de recherche français ou étrangers, des laboratoires publics ou privés. 


\title{
Subwavelength seismic metamaterial with an ultra-low frequency band gap
}

\author{
Yi Zeng ${ }^{1,2,3}$, Pai Peng ${ }^{2}$, Qiu-Jiao Du ${ }^{2, *}$,Yue-Sheng Wang ${ }^{1, *}$ and Badreddine Assouar ${ }^{3, *}$ \\ ${ }^{1}$ Department of Mechanics, School of Mechanical Engineering, Tianjin University, Tianjin 300350, \\ China \\ ${ }^{2}$ School of Mathematics and Physics, China University of Geosciences, Wuhan 430074, China \\ ${ }^{3}$ Institut Jean Lamour, CNRS, University de Lorraine, Nancy 54506, France \\ * Electronic mail: qiujiaodu@cug.edu.cn (Q.-J. Du), yswang@tju.edu.cn (Y.-S. Wang), \\ badreddine.assouar@univ-lorraine.fr (B. Assouar).
}

\begin{abstract}
A subwavelength seismic metamaterial (SMs) consisting of a three-component SM plate (SMP) and a half space is proposed to attenuate ultra-low frequency seismic surface waves. The design concept and models are verified firstly by lab-scale experiments on the SM consisting of a two-component SMP and a half space. Then we calculate the band structures of the one-dimensional and two-dimensional subwavelength SMs, and evaluate their ability to attenuate Rayleigh waves. A wide ultra-low frequency band gap can be found. And the Rayleigh waves are deflected by the subwavelength SM and converted into bulk waves in the frequency range of this band gap. When the number of the unit cells of the subwavelength SM is sufficient, the transmission distance and deflection angle of the Rayleigh waves are constant at the same frequency. This discovery is expected to open up the possibility of pragmatic
\end{abstract}


seismic protection for large nuclear power plants, ancient buildings and metropolitan areas.

Key words: seismic metamaterials; ultra-low frequency band gap; surface waves; Rayleigh waves; three-component structures 


\section{Introduction}

Millions of earthquakes occur every year on the planet we live in. ${ }^{1}$ According to the statistics of China Earthquake Networks Center, there have been 836 major earthquakes of magnitude 6 and above in the past 100 years (1919-2018), i.e. an average of more than eight times a year. The major casualties caused by Earthquakes are due to the collapse of buildings. The destruction of some important buildings and facilities will bring devastating disasters and irreparable damage, such as nuclear power plants and ancient buildings. The seismic metamaterials $(\mathrm{SMs}){ }^{2-5}$ proposed in recent years can prevent these things in a clever way.

SMs with periodically arranged structures have band gaps (BGs). ${ }^{6-9}$ In the frequency ranges of the BGs, the seismic waves cannot pass through the SMs. In 2014, an experiment with cylindrical holes arranged periodically verified that this SM can attenuate seismic waves in the BG. ${ }^{3}$ When the shape of the holes is changed or some special material is filled in the holes, the BG characteristics and the attenuation effect of the SMs on the seismic waves can still be found. ${ }^{10-12}$ In addition, the research on SMs based on half-space conditions has also received much attention. It has been found that the forest can attenuate the seismic surface waves, because these trees in the forest, which can be modeled as pillars over a half space, can open the BG of the surface waves. ${ }^{13}$ Therefore, various pillars placed on the ground have been extensively studied and experimentally verified. ${ }^{14-17}$ It is worth mentioning that the use of Matryoshka-like structures, ${ }^{18}$ fractal structures ${ }^{19,20}$ and "rainbow trapping effect" ${ }^{4}$ can realize the attenuation of seismic surface waves over a wider range of 
frequency. Among them, Miniaci et al. ${ }^{20}$ demonstrated the possibility of lowering BGs in elastic systems by introducing self-similar structures at multiple scales experimentally. In addition, some multi-component SMs have been proposed to attenuate the seismic bulk waves and surface waves. ${ }^{2,21-27}$ However, the SMs, which are often made of a very soft coating and a very heavy core, ${ }^{10}$ can attenuate seismic waves below $5 \mathrm{~Hz}$ which contains the resonant frequencies of many large buildings and metropolises. There are also some works ${ }^{28,29}$ that consider the effectiveness of SMs in layered soils. It is consistent with the fact that the substrate in practice is composed of multiple layers of soil. ${ }^{30}$ These works will facilitate the practical applications of SMs. Although SMs clamped by bedrock have a zero-frequency BG, they are only suitable for areas where the embedded depth of bedrock is shallow. ${ }^{29,31}$ In this paper, a subwavelength $\mathrm{SM}$ consisting of three-component seismic metamaterial plate (SMP) on a half-space is proposed to attenuate ultra-low frequency seismic surface waves in a variety of geological situations. We first review the surface wave transmission experiments of the two-component SM composed of two-component SMP on a half-space. ${ }^{32}$ This is the experimental basis of the three-component SMs in this paper. Then we calculate the band structures of the one-dimensional (1D) and two-dimensional (2D) SMs, check their attenuation ability to Rayleigh waves, and specifically discuss the propagation of Rayleigh waves in the $\mathrm{SM}$ in the range of the ultra-low frequency BG (UFBG). The subwavelength SM does not need bedrock or fixed boundary condition to clamp the bottom of the pillars metamaterials. This explains that the UFBG and the wide BG can be more universally 
implemented theoretically than the previous works. ${ }^{29,31}$ And the subwavelength SM uses traditional building materials, making it a very practical design.

\section{Methods}

\subsection{Numerical simulations}

In this paper, we use Solid Mechanics Module of COMSOL Multiphysics to calculate the band structures and the propagation of Rayleigh waves in the SM. The meshes are free triangular mesh with quadratic serendipity element for $2 \mathrm{D}$ models, free tetrahedral mesh with quadratic Lagrange element for 3D models. The minimum wavelength involved in this work is approximately $5.6 \mathrm{~m}(3.7 a)$ when the frequency is $22 \mathrm{~Hz}$. In simulation, the maximum sizes of the mashes is set to $a / 5$, which is about $1 / 18$ of the minimum wavelength. Therefore, the meshes are small enough to get the converge results. ${ }^{33,34}$ For the one-dimensional periodic SM, the reduced wave vector from 0 to $\pi / a$ ( $a$ is the period of the $\mathrm{SM}$ ) is used to obtain the eigenvalues ${ }^{35}$. For the two-dimensional periodic SM, which is highly symmetrical, the wave vector along $\Gamma$ $-X$ direction ${ }^{36}$ is used to calculate the band structures. We mainly target Rayleigh waves which are often concerned in the study of SMs as they can travel far away and are extremely harmful to buildings in the mid- and far-fields. So the excitation condition in the frequency domain calculation is mainly used to obtain Rayleigh waves, which has sagittal displacement with a maximum value of $0.01 \mathrm{~m}$ both in the $x$ and $z$ directions on the surface. ${ }^{4,17}$ When we calculate the attenuation ability of SMs, the transmission spectrum is defined as $T S=20 \times \log _{10}\left(A_{1} / A_{0}\right)$ where $A_{1}$ is the acceleration of a point behind the SM, and $A_{0}$ is the acceleration of the same point 
when there is no SM. Therefore, the amplitude of the excitation does not change the value of $T S$ in the simulation calculation and the attenuation of seismic waves by SM is under linear elastic conditions.

Figure 1(a) shows that the unit cell of the two-component SMP consists of a steel core wrapped with a flexible foam plate. Figure 1(b) shows the unit cell of the two-component SM consisting of the two-component SMP and half space. The band structures of two-component SMP and SM are calculated along $\Gamma-X$ direction, which are shown in Figs. 1(c) and 1(d) respectively.

\subsection{Lab-scale experimental setup}

In the experiment, the sample comprising foam-steel composite is zoomed in the upper right corner of Fig. 1(e). The size of the steel core is $140 \mathrm{~mm} \times 40 \mathrm{~mm} \times 40$ $\mathrm{mm}$, and the thickness of the flexible foam plate is $5 \mathrm{~mm}$. The size of the glass box is $1.2 \mathrm{~m} \times 0.5 \mathrm{~m} \times 0.5 \mathrm{~m}$, and the material of the substrate is sand. In order to reduce the reflection of the elastic wave by the glass wall, pebbles are placed around the wall. ${ }^{24}$ The details of the experimental instruments used in the present work are consistent with this reference ${ }^{32}$. A thick layer of high-damping plastic foam is placed under the glass box to achieve a similar effect to the vibration isolation platform.

The accelerations are carried out by using two accelerometers placed on two symmetrical points (labels D and E in Fig. 1(e)). One (label D) is placed in the free zone where there is no interaction between the SM and the seismic wave, and the other $(($ label E)) is located behind the SM. Suppose that the acceleration values of points $\mathrm{D}$ and $\mathrm{E}$ are $A_{\mathrm{D}}$ and $A_{\mathrm{E}}$, respectively. Then the attenuation spectrum of $\mathrm{SM}$ on 
the surface wave is $20 \times \log _{10}\left(A_{\mathrm{E}} / A_{\mathrm{D}}\right)$ shown in Fig. 1(f).

\section{Discussion of models}

\subsection{Experimental verification of numerical model of two-component SM}

In order to verify if the new design of SMs can attenuate seismic surface waves, we have performed lab-scale experiment for a supercell of eight unit cells (four rows) of two-component SM to measure the transmission spectrum. The geometric parameters are: $a=5 \mathrm{~cm}, b=1 \mathrm{~cm}, c=4 \mathrm{~cm}, h=15 \mathrm{~cm}$ and $H=20 \mathrm{a}$. The material parameters ${ }^{32}$ are illustrated in Table 1. All parameters used in the simulation are consistent with those in the lab-scale experiment.

Comparing the two band structures, we can find that when the SMP is placed on the half space, the original band is basically unchanged, but the sound cone (dark gray area in Fig. 1(d)) is added due to the existence of the half space. So, we can not only keep the BG of the SMP itself in Fig. 1(d). It is found that the addition of the substrate does not affect the original band structure of SMP. That is to say, the band structure of the SM composed of the two-component SMP and a half space is almost equal to band structure of the two-component SMP plus the sound cone. The sound cone, the boundary of which is formed by the slowest bulk wave propagating in the substrate, can be used to immediately identify real propagating surface waves, bulk modes as well as leaky surface modes and spurious PML modes. ${ }^{37,38}$ Therefore, the wide BG of the SMP itself is preserved. At $110 \mathrm{~Hz}-300 \mathrm{~Hz}$, we can verify that the SM can attenuate the surface waves well by lab-scale experiments and simulated calculations of the supercell, which corresponds to the BG in Fig. 1(d). The geometric model, 
material parameters, and position of the supercell and substrate used in the simulation calculations are consistent with the lab-scale experiments. In addition, the boundary formed by the glass wall is replaced by a low reflection boundary to reduce reflected waves.

This lab-scale experiment demonstrates that the two-component SM can attenuate the surface waves in the frequency range of the BG well, but this SM is powerless for the lower frequency surface waves. However, our previous work ${ }^{32}$ shows that this SM composed of SMP and substrate can strongly attenuate surface waves in numerical simulation and lab-scale experiments. In addition, this kind of SM only needs to be laid on the base layer, which reduces costs and simplifies construction. The functional shortcomings of the two-component SM prompt us to further improve the SM to possess the above-mentioned advantages and also attenuate surface waves at ultra-low frequencies. In the following, the effect of SM composed of the three-component SMP and substrate is systematically investigated on the attenuation of surface waves in the ultra-low frequency range.

\subsection{Discussions of the SM}

From the above simulations and lab-scale experiment of the SM consisting of the two-component SMP and half space, it can be seen that the band structure of the $\mathrm{SM}$ is almost equal to the band structure of the SMP plus the sound cone. This SM can effectively attenuate the seismic surface waves to protect the buildings. Next, we will use FEM calculations to discuss the band structure and attenuation effect of the subwavelength SM consisting of the three-component SMP and a half space on the 
seismic surface waves. The UFBG of the subwavelength SM is discussed under the one-dimensional (1D) and two-dimensional (2D) periodic models.

\subsubsection{Model of 1D SM}

Figure 2(a) shows a unit cell of 1D three-component SMP, which is set to a Bloch periodic boundary condition in the $x$ direction and a free boundary condition in the $z$ direction. Figure 2(b) shows a unit cell of the 1D SM consists of a unit cell of 1D three-component SMP and a half space. It is set to the Bloch period boundary condition in the $x$ direction, the upper boundary is a free boundary, and the lower boundary is a fixed boundary. The geometric parameters are: $a=1.5 \mathrm{~m}, a_{1}=0.6 a, a_{2}$ $=0.5 a, H=20 a$. The material parameters are illustrated in Table $1 .{ }^{11}$

Figure 2(c) shows a schematic diagram of the system used to calculate the transmission spectrum. Since the UFBG of the subwavelength SM is mainly studied and discussed in this paper, and the wavelength of the seismic surface wave is large in the range of the UFBG, the values of the geometric parameters involved here are all large, especially the depth $(500 a)$ of the substrate, the distance (340a) from the source (point A in Fig. 2(c)) to the ULSM and the number (1600) of the unit cells of the subwavelength SM. Point B (Fig. 2(c)) is the signal collection point. Suppose that the acceleration at point $\mathrm{B}$ located after the SMP is $A_{1}$, and the acceleration at point $\mathrm{B}$ without the SMP is $A_{0}$. Then the transmission function is defined as $20 \times \log _{10}\left(A_{1} / A_{0}\right)$. To prevent unwanted reflections, we add a perfectly matched layer (PML) with a thickness of $50 a$ which is about the same as the incoming wavelength at low frequencies, as shown in Fig. 2(c). Among them, the PML scaling factor and the PML 
scaling curvature parameter are 1 . Some works ${ }^{12,39}$ also use low reflection boundaries to achieve similar results.

\subsubsection{Band structure and transmission spectrum of 1D SM}

For a 1D three-component SMP based on local resonance, a BG can easily occur. As shown in Fig. 3(a), a narrow BG appears in the range of $10.0-14.0 \mathrm{~Hz}$. Even if the unit cells in Fig. 2(a) are periodically arranged in two dimensions, the BG is very narrow. ${ }^{22}$ It is conceivable that such a narrow BG cannot meet our needs. However, when we place this SMP on a half space (i.e. the SM), we are surprised to find two wide BGs $(0-9.0 \mathrm{~Hz}$ and $9.4-22.0 \mathrm{~Hz})$ for surface waves. And one of them is a UFBG. By comparing the band structures in Figs. 3(a) and 3(b), we can easily find that the band structure of the 1D SM consisting of the 1D three-component SMP and a half space is almost equal to the band structure of the 1D three-component SMP plus the sound cone. Therefore, the UFBG and the wide BG are generated shown in Fig. 3(b). It is worth noting that there are still bulk modes in the frequency range of the UFBG without any surface mode. This result proves that this work is completely different from the previous ones ${ }^{29,31}$ which do not have modes in the UFBG. In Fig. 3(d), the effective density, shear modulus and bulk modulus of the SMP are obtained by using numerical methods. ${ }^{40}$ We can see that the BG of SMP is due to the appearance of its negative effective mass density.

Through the transmission spectrum of the subwavelength SM, we can also see the existence of these two wide BGs. In the range of the UFBG, we see that the seismic surface waves are well attenuated in the range of $0.5-9.0 \mathrm{~Hz}$. However, for the 
seismic surface waves close to zero frequency, the attenuation effect needs to be improved. Because the number of unit cells in the proposed subwavelength SM is infinite because of the periodic boundary condition when calculating the band structure, while the number of the unit cells in the subwavelength SM is finite (1600 unit cells) when calculating the transmission spectrum. Therefore, we hypothesize that the number of the unit cells of the ULSM and the lower limit of the attenuation zone in the transmission spectrum are closely related.

\subsubsection{Discussion on the UFBG of 1D SM}

To discover the relationship between the number of the unit cells of the subwavelength SM and the lower limit of the attenuation zone in the transmission spectrum, we simulate and plot the displacements of surface waves of the system varying with the frequency by calculating the transmission spectrum. As shown in Fig. 4(a), we use the subwavelength SM of 1600 unit cells placed from $0 \mathrm{~m}$ to $2400 \mathrm{~m}$ for calculation. The transmission distance of the Rayleigh waves increases in the subwavelength SM exponentially with the decrease of frequencies from $5.0 \mathrm{~Hz}$ to around $0.5 \mathrm{~Hz}$. It is found that in a higher frequency range between $3 \mathrm{~Hz}$ to $5 \mathrm{~Hz}$, we can also attenuate seismic surface waves with very few seismic metamaterial units.

When the frequency is less than $0.5 \mathrm{~Hz}$, Rayleigh waves pass through the 1600 unit cells of subwavelength SM. We can speculate that if the number of unit cells in the subwavelength SM increases, the propagation of the lower-frequency Rayleigh waves can be attenuated. Physically speaking, this subwavelength SM is a novel SM with the UFBG. For practical applications, it is also very convenient to select the number of 
unit cells according to the frequency range that we consider. We have studied the transmission of Rayleigh waves in the subwavelength SM at $2.2 \mathrm{~Hz}$ to demonstrate the mechanism of the subwavelength SM. As shown in Fig. 4(c), when the Rayleigh waves enter the subwavelength SM, they are deflected at a certain angle and converted into bulk waves. As shown in Figs 4(c) and 4(d), the waves at the left end of the SM are Rayleigh waves. The energy of Rayleigh waves is mainly concentrated in the depth of two wavelengths below the surface. The Rayleigh waves are converted into bulk waves in the SM, because there is no surface wave mode in the SM at the frequency of the UFBG, but the bulk wave modes exist.

To explain this phenomenon, we draw a dotted line with the frequency of $2.2 \mathrm{~Hz}$ on the band structure of the subwavelength SM shown in Fig. 4(b). At $2.2 \mathrm{~Hz}$, there is no surface wave mode in the subwavelength SM. However, there are a large number of bulk wave modes outside the sound cone. That is to say, at $2.2 \mathrm{~Hz}$, only the bulk wave modes exist in the subwavelength SM. Therefore, Rayleigh waves will be deflected and converted into bulk waves as shown in Fig. 4(c). This principle is also used to achieve low-consumption energy transmission and other functions in topological metamaterials and has been validated in experiments. ${ }^{41,42}$

We also use the subwavelength $\mathrm{SM}$ of 100 unit cells placed from $0 \mathrm{~m}$ to $150 \mathrm{~m}$ with for the calculation of the transmission spectrum in Fig. 4(d). We find that the deflection of Rayleigh waves is almost the same as the subwavelength SM with 1600 unit cells. When the Rayleigh waves propagate to the position of $X=60 \mathrm{~m}$ (presumably at the position of the 40th unit cell), most of the energy has turned from 
the surface to the bulk below the surface. This also shows that when the number of the unit cells of the subwavelength SM is sufficient, the transmission distance and deflection angle of the Rayleigh waves in the subwavelength SM are constant at the same frequency. In practical applications, the method of arranging the number of the unit cells of the subwavelength SM according to the specified frequency range is effective.

In order to investigate the influence of the material parameters of the substrate on the UFBG, we consider the effect of the Young's modulus and mass density of the substrate on the upper frequency bounds of the UFBG for the 1D subwavelength SM. The material parameters of the substrate are selected within the actual soil parameters 19. However, when the Young's modulus of the substrate increases from $10 \mathrm{MPa}$ to $100 \mathrm{MPa}$, and the mass density of the substrate is between $1600 \mathrm{~kg} / \mathrm{m}^{3}$ and 2200 $\mathrm{kg} / \mathrm{m}^{3}$, the upper boundary $(9.0 \mathrm{~Hz})$ of the UFBG does not change. In this range of the Young's modulus and the mass density, the upper boundary of the UFBG is determined by the band of the local resonance of the SMP, which is invariable. From the effect of the Young's modulus and mass density of the substrate on the upper frequency bounds of the UFBG for the 1D SM, we can find that the subwavelength $\mathrm{SM}$ is suitable for different soils in different regions. This also shows that the subwavelength SM is universal.

The values for material parameters of the substrate are swept and are intended for qualitative comparison only ${ }^{40}$. Through the simulation calculation and discussion, we find this important universal equation: the band structure of the SM composed of the 
SMP and a half space is almost identical to band structure of the SMP plus the sound cone. It is noteworthy that this conclusion is incorrect when the heavy core of the unit cell of the SMP connects the substrate. ${ }^{10,11}$

The above 1D SWSM can be modelled as a two-layered medium as shown in Fig. 5(a). The green part is the effective medium of the SMP. Its upper boundary is the free boundary, and the thickness is $a=1.5 \mathrm{~m}$. In the low frequency region (0 - $5 \mathrm{~Hz})$ away from local resonance, as shown in Fig. 3(d), the effective mass density of the SMP is about $2000 \mathrm{~kg} / \mathrm{m}^{3}$. And the effective Young's modulus $E_{\mathrm{e}}=27.12 \mathrm{GPa}$, Poisson's ratio $v_{\mathrm{e}}=0.3$. The substrate shown in Fig. 5(a) is a half-space of soil.

For the layered medium shown in Fig. 5(a), the dispersion curve of the Rayleigh wave can be calculated by the fast scalar method. ${ }^{43}$ As shown in Fig. 5(b), the dispersion curve of the layered medium only supports a single waveguide mode and exhibits a typical inverse dispersion. ${ }^{44}$ The wave velocity of Rayleigh waves increases with the frequency increasing in the two-layered medium in the range of $0-0.0125 \mathrm{~Hz}$. And there is a cut-off frequency $(0.0125 \mathrm{~Hz})$. It means that, when the frequency is greater than the $0.0125 \mathrm{~Hz}$, Rayleigh waves will not exist in the effective two-layered medium. This also verifies the correctness of the UFBG because $0.0125 \mathrm{~Hz}$ is close to zero.

In practice, the substrates are composed of multiple layers of soil. ${ }^{28-30}$ For illustration, we divide the substrate into three layers of soil. ${ }^{29}$ Each layer has a thickness of $10 \mathrm{~m}$ as shown in Fig. 6(a). Each layer of soil has an identical Poisson's ratio of 0.3 and a density of $1800 \mathrm{~kg} / \mathrm{m}^{3}$. The Young's modulus from the top layer to the bottom layer is 
5, 100 and $2000 \mathrm{MPa}$. The values for material parameters of the substrate are intended also for qualitative comparison only. ${ }^{40}$ The UFBG still exists and the width is unchanged as shown in Fig. 6(b). This shows that even if the substrate is composed of multiple layers of soil, this SM can still attenuate ultra-low frequency seismic surface waves.

\subsubsection{Model of 2D SM}

In order to verify that the above the subwavelength SM still has a UFBG in the three-dimensional space, we have studied the 2D SM as shown in Fig. 7(b). Similarly, we also calculate the band structure of the unit cell of the 2D three-component SMP shown in Fig. 7(a). The unit cell of the 2D SM consists of a unit cell of the 2D three-component SMP and a half space. The geometric parameters $\left(a, a_{1}, a_{2}\right.$ and $\left.H\right)$ and the material parameters of the four materials (concrete, rubber, steel and soil) are consistent with those of the 1D SM. We set the Bloch period boundary conditions on the four vertical boundaries of the 2D SMP; and the upper and lower boundaries are free. The Bloch period boundary condition is set on the four vertical boundaries of the 2D SM; the lower boundary is set as the fixed boundary condition, and the upper boundary is set as the free boundary condition. The system used for transmission spectrum calculation is similar to Fig. 2(c), so it is not illustrated here. ${ }^{17}$

\subsubsection{Band structure and transmission spectrum of 2D SM}

Metamaterials based on the local resonance is a subject of debate and discussion. Although they can obtain BGs at low frequencies, the width of the BGs needs to be expanded. ${ }^{38,45,46}$ As shown in Fig. 8(a), the BG of the 2D three-component SMP is 
very narrow and only exists around $10.0-12.0 \mathrm{~Hz}$. Even if the unit cells of such three-component SMP are three-dimensionally periodically arranged, the width of the BG is also narrow. ${ }^{23}$ However, the 2D SM has two wide BG for surface waves, and one of them is an ultra-low BG. By comparing the band structures in Figs. 8(a) and (b), we can easily find that the band structure of the subwavelength SM consisting of the 2D three-component SMP and a half space is almost equal to the band structure of the 2D three-component SMP plus the sound cone. This is also the reason for the wide ultra-low BG.

We calculate the transmission spectrums of Rayleigh waves in the $1 \mathrm{D}$ and $2 \mathrm{D} \mathrm{SMs}$ (along $\Gamma X$ direction) with 100 unit cells at $0.5-5.0 \mathrm{~Hz}$ which is within the UFBG. The attenuation effect of the two kinds of subwavelength SM is almost the same, and the 2D ULSM is slightly better than the $1 \mathrm{D} \mathrm{SM}$ at low frequencies $(0.5-4.0 \mathrm{~Hz})$. This shows that the 2D SM can also attenuate ultra-low frequency surface waves in the three-dimensional space.

\subsubsection{Discussion on the UFBG of 2D SM}

As shown in Fig. 9, by comparing the propagations of Rayleigh waves in the 1D and 2D SMs (xz section diagram) with 100 unit cells at 4.0 Hz, the Rayleigh waves in the 2D SM also convert from surface waves to bulk waves, and the deflection angle of the Rayleigh wave is almost the same as that of the 1D SM. This shows that the 2D SM can attenuate surface waves in the frequency range of its UFBG to protect the target building. Its mechanism is consistent with that of the 1D SM.

\section{Conclusion}


In summary, through the lab-scale experiments and numerical simulation of the SM consisting of the two-component SMP and a half space, we demonstrate this new design methodology to create SMs to attenuate seismic surface waves. In addition, lab-scale experiments are also the important basis for the application of three-component subwavelength SM. Then we propose a three-component subwavelength SM consisting of the three-component SMP and a half space. By investigating the influence of the material parameters of the substrate on the 1D SM, we find that the band structure of the SM composed of the SMP and a half space is almost equal to the band structure of the SMP plus the sound cone. By analyzing the propagation of Rayleigh waves in the 1D and 2D SMs in the frequency range of the UFBG, we find that Rayleigh waves are deflected at a certain angle and converted into bulk waves. When the number of the unit cells of the subwavelength SM is sufficient, the transmission distance and deflection angle of the Rayleigh waves in the subwavelength SM are constant at the same frequency. In summary, the SM consisting of only one layer of the SMP buried underground and substrate has an ultra-low frequency BG and can convert Rayleigh waves into bulk waves in the frequency range of the BG. Meanwhile, the simple design of this SM is conducive to its manufacturing and practical application and the efficiency of the SM to attenuate Rayleigh waves is also very significant. All the phenomena demonstrate that the subwavelength SM can attenuate surface waves in the frequency range of its UFBG to protect the target building. This subwavelength SM is expected to protect large nuclear power plants, ancient buildings and metropolises from damage by seismic 
waves. This paper not only demonstrates the application prospects of subwavelength

$\mathrm{SM}$, but also provides design guidance for surface wave researchers.

\section{Data Availability Statement}

The data that support the findings of this study are available from the corresponding author upon reasonable request.

\section{Acknowledgments}

We thank the National Natural Science Foundation of China (NNSFC) under Grant Nos. 41974059 and 11702017, the Key Program of the National Natural Science Foundation of China (NNSFC) under Grant No. 41830537, and the National Programme on Global Change and Air-Sea Interaction (GASI-GEOGE-02).

\section{References}

R. K. Reitherman, in Earthquakes and engineers: an international history, 2012 (American Society of Civil Engineers).

2 G. Finocchio, O. Casablanca, G. Ricciardi, U. Alibrandi, F. Garescì, M. Chiappini, and B. Azzerboni, Applied Physics Letters 104, 191903 (2014).

3 S. Brûlé, E. Javelaud, S. Enoch, and S. Guenneau, Physical review letters 112, 133901 (2014).

4 A. Colombi, D. Colquitt, P. Roux, S. Guenneau, and R. V. Craster, Scientific reports 6, 27717 (2016).

5 Y.-F. Wang, Y.-Z. Wang, B. Wu, W. Chen, and Y.-S. Wang, Applied Mechanics Reviews 72 (2020).

6 F. Meseguer, M. Holgado, D. Caballero, N. Benaches, J. Sánchez-Dehesa, C. López, and J. Llinares, Physical Review B 59, 12169 (1999).

7 V. Laude, M. Wilm, S. Benchabane, and A. Khelif, Physical Review E 71, 036607 (2005).

8 T.-T. Wu, L.-C. Wu, and Z.-G. Huang, Journal of Applied Physics 97, 094916 (2005).

$9 \quad$ A. Khelif, B. Aoubiza, S. Mohammadi, A. Adibi, and V. Laude, Physical Review E 74, 046610 (2006). (2016). 
Q. Du, Y. Zeng, G. Huang, and H. Yang, AIP Advances 7, 075015 (2017).

X. Pu and Z. Shi, Soil Dynamics and Earthquake Engineering 98, 67 (2017).

A. Colombi, P. Roux, S. Guenneau, P. Gueguen, and R. V. Craster, Scientific reports 6, 19238 (2016).

Y.-f. Liu, J.-k. Huang, Y.-g. Li, and Z.-f. Shi, Construction and Building Materials 199, 737 (2019).

P. Roux, D. Bindi, T. Boxberger, A. Colombi, F. Cotton, I. Douste - Bacque, S. Garambois, P. Gueguen, G. Hillers, and D. Hollis, Seismological Research Letters 89, 582 (2018).

D. Colquitt, A. Colombi, R. Craster, P. Roux, and S. Guenneau, Journal of the Mechanics and Physics of Solids 99, 379 (2017).

Y. Zeng, Y. Xu, K. Deng, Z. Zeng, H. Yang, M. Muzamil, and Q. Du, Journal of Applied Physics 123, 214901 (2018).

Y. Zeng, Y. Xu, H. Yang, M. Muzamil, R. Xu, K. Deng, P. Peng, and Q. Du, International Journal of Solids and Structures 185-186, 334 (2020).

Q. Du, Y. Zeng, Y. Xu, H. Yang, and Z. Zeng, Journal of Physics D: Applied Physics 51, 105104 (2018).

M. Miniaci, A. Krushynska, A. S. Gliozzi, N. Kherraz, F. Bosia, and N. M. Pugno, Physical Review Applied 10, 024012 (2018).

O. Casablanca, G. Ventura, F. Garescì, B. Azzerboni, B. Chiaia, M. Chiappini, and G. Finocchio, Journal of Applied Physics 123, 174903 (2018).

Y. Yan, A. Laskar, Z. Cheng, F. Menq, Y. Tang, Y. Mo, and Z. Shi, Journal of Applied Physics 116, 044908 (2014).

Y. Yan, Z. Cheng, F. Menq, Y. L. Mo, Y. Tang, and Z. Shi, Smart Material Structures 24 (2015).

S. Krödel, N. Thomé, and C. Daraio, Extreme Mechanics Letters 4, 111 (2015).

Z. Cheng and Z. Shi, Earthquake Engineering \& Structural Dynamics 47, 925 (2018).

N. Aravantinos-Zafiris and M. Sigalas, Journal of Applied Physics 118, 064901 (2015).

A. Palermo, S. Krödel, A. Marzani, and C. Daraio, Scientific reports 6, 39356 (2016).

X. Pu and Z. Shi, Construction and Building Materials 180, 177 (2018).

Y. Chen, Q. Feng, F. Scarpa, L. Zuo, and X. Zhuang, Materials \& Design, 107813 (2019).

G. Gao, N. Li, and X. Gu, Soil Dynamics and Earthquake Engineering 69, 251 (2015).

Y. Achaoui, T. Antonakakis, S. Brûlé, R. V. Craster, S. Enoch, and S. Guenneau, New Journal of Physics 19, 063022 (2017).

Y. Zeng, Y. Xu, K. Deng, P. Peng, H. Yang, M. Muzamil, and Q. Du, Journal of Applied Physics 125, 224901 (2019).

L. De Marchi, A. Marzani, and M. Miniaci, NDT \& E International 54, 115 (2013).

F. Moser, L. J. Jacobs, and J. Qu, Ndt \& E International 32, 225 (1999).

B. Graczykowski, F. Alzina, J. Gomis-Bresco, and C. Sotomayor Torres, Journal of Applied Physics 119, 025308 (2016).

F. Maurin, C. Claeys, E. Deckers, and W. Desmet, International Journal of Solids and Structures (2017).

M. Molerón, S. Félix, V. Pagneux, and O. Richoux, Journal of Applied Physics 111, 114906 (2012).

Y. Achaoui, A. Khelif, S. Benchabane, L. Robert, and V. Laude, Physical Review B 83, 104201 (2011).

J. Huang, Z. Shi, and W. Huang, Physica B: Condensed Matter 516, 48 (2017). 
41 J. Lu, C. Qiu, W. Deng, X. Huang, F. Li, F. Zhang, S. Chen, and Z. Liu, Physical review letters 120, 116802 (2018). (2019).

43 B. Zhang, M. Yu, C.-Q. Lan, and W. Xiong, The Journal of the Acoustical Society of America 100, 3527 (1996).

T. Yang, Forward Modeling of Zigzag Dispersion and Pavement Systems Dispersion Curves, Thesis, Central South University 2004.

45 Z. Liu, X. Zhang, Y. Mao, Y. Zhu, Z. Yang, C. Chan, and P. Sheng, Science 289, 1734 (2000).

46 P. Sheng, X. X. Zhang, Z. Liu, and C. T. Chan, Physica B: Condensed Matter 338, 201 (2003). 


\section{Figure captions}

Figure 1 (a) The unit cell of two-component SMP. (b) The unit cell of two-component SM consisting of two-component SMP and half space. Note that the yellow, purple and black areas represent soil, flexible foamed plate and steel core, respectively. Band structures of (c) two-component SMP and (d) two-component SM. (e) Experimental setup and the design of unit cell. (f) Experimental and numerical transmission spectrums for supercell of eight unit cells (four rows) of two-component SM.

Figure 2 (a) The unit cell of the 1D three-component SMP. (b) The unit cell of 1D SM. (c) A schematic diagram of a system for calculating transmission spectra. Note that the yellow, gray, purple and black areas represent soil, concrete, rubber and steel core, respectively.

Figure 3 (a) Band structure of the 1D three-component SMP. (b) Band structure of the 1D SM. (c) Transmission spectrum of the system in Fig. 2(c). (d) The effective parameters of the 1D three-component SMP.

Figure 4 (a) The displacements of surface waves of the system varying with the frequency by calculating the transmission spectrum. (b) subwavelength SM's band structure and a dotted line with a frequency of $2.2 \mathrm{~Hz}$. (c) The propagation of Rayleigh waves in subwavelength SM with 1600 unit cells at $2.2 \mathrm{~Hz}$. (d) The propagation of Rayleigh waves in subwavelength SM with 100 unit cells at $2.2 \mathrm{~Hz}$. The colors indicate the total displacement $(u)$ ranging from 0 to maximum. 
Figure 5 (a) Effective two-layered medium of 1D SM, (b) Rayleigh waves dispersion curve of the two-layered media.

Figure 6 (a) Schematics of the unit cell and (b) band structure of the proposed SMP on three layers of soil.

Figure 7 (a) The unit cell of the 2D three-component SMP. (b) The unit cell of 2D SM. Note that the yellow, gray, purple and black areas represent soil, concrete, rubber and steel core, respectively.

Figure 8 (a) Band structure of the 2D three-component SMP. (b) Band structure of the 2D SM. (c) Transmission spectrums of 1D and 2D subwavelength SMs (SWSMs) with 100 unit cells in the frequency range of $0.5 \mathrm{~Hz}-5.0 \mathrm{~Hz}$.

Figure 9 The propagations of Rayleigh waves in (a) 1D SM and (b) 2D SM ( $x z$ section diagram) with 100 unit cells at $4.0 \mathrm{~Hz}$. The colors indicate the total displacement $(u)$ ranging from 0 to maximum. 


\section{Figures}
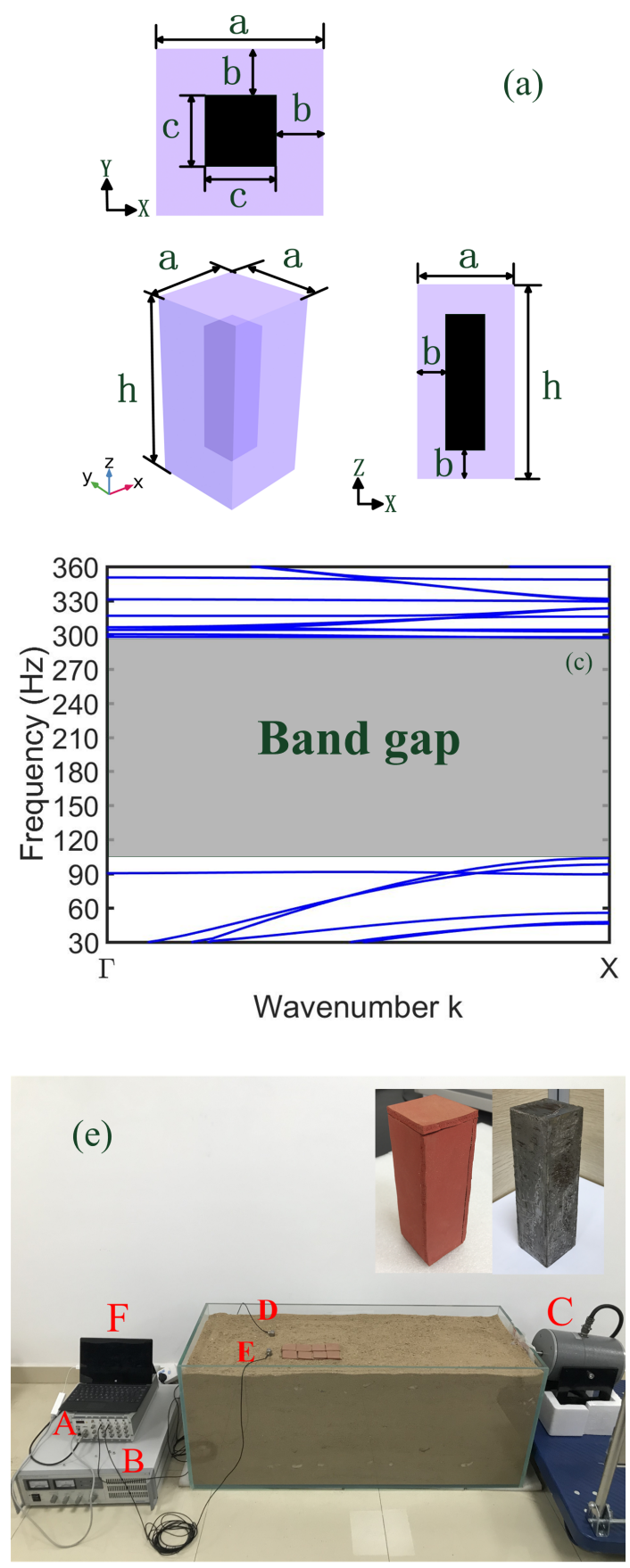

(b)
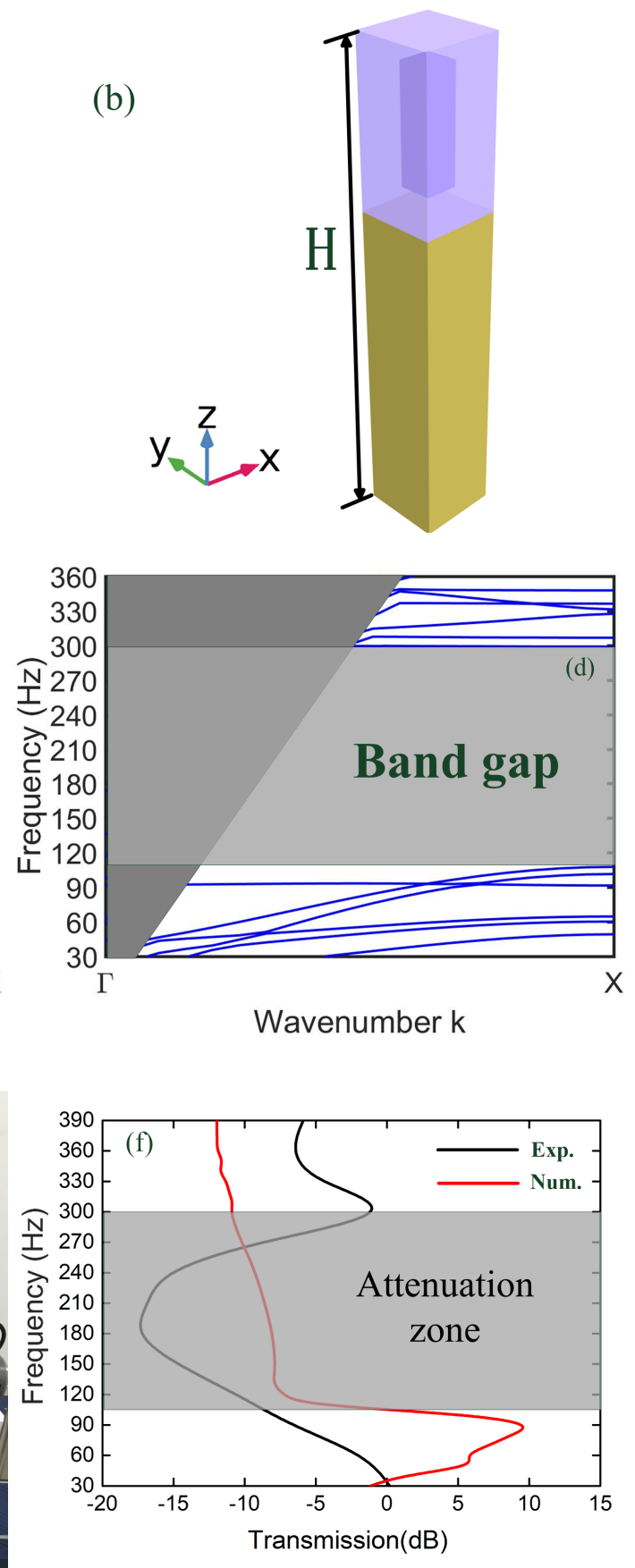

Figure1 


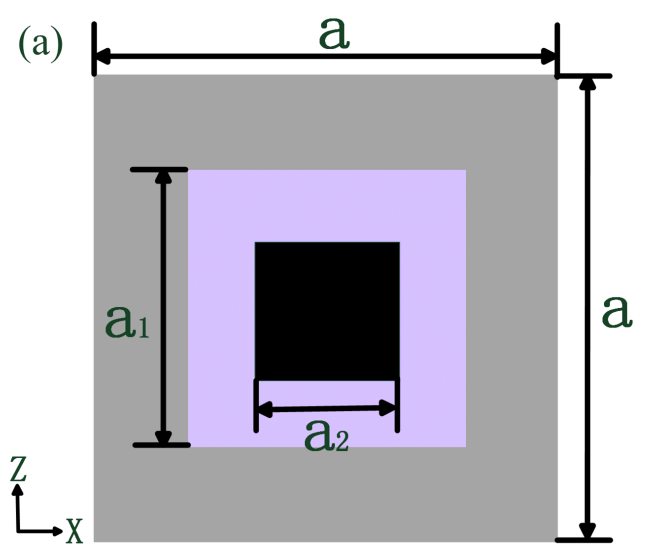

(b)
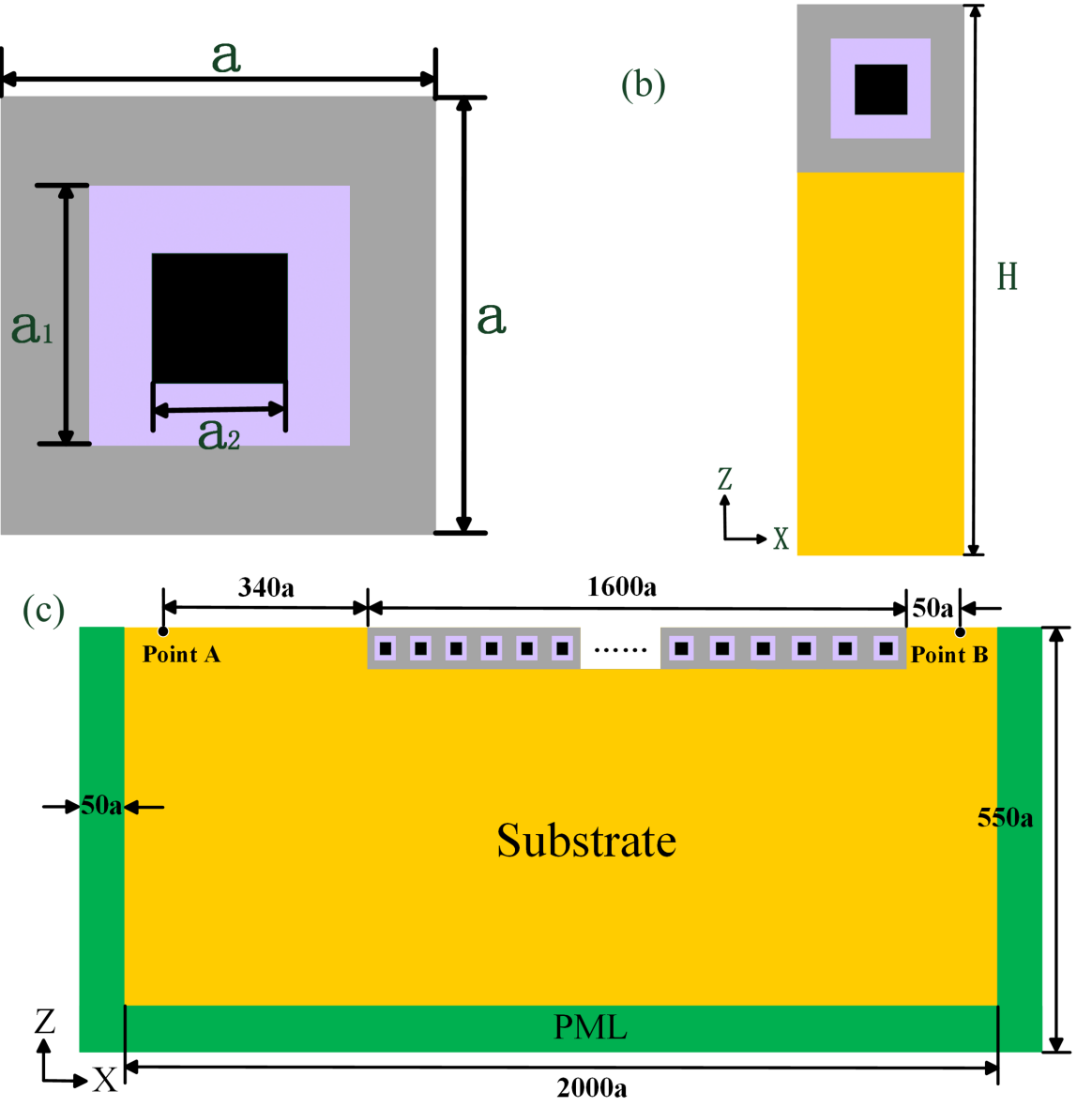

Figure2 

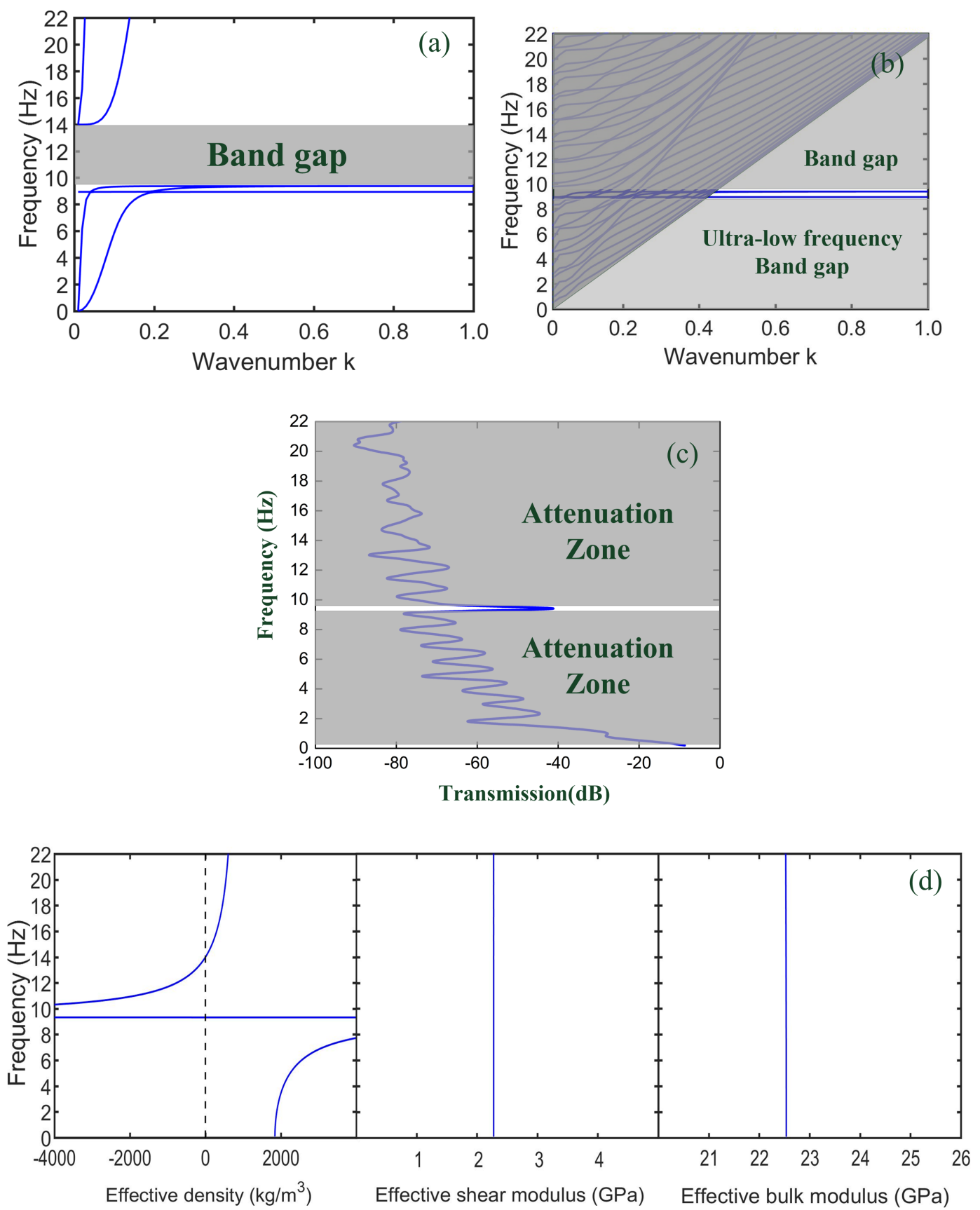

Figure3 

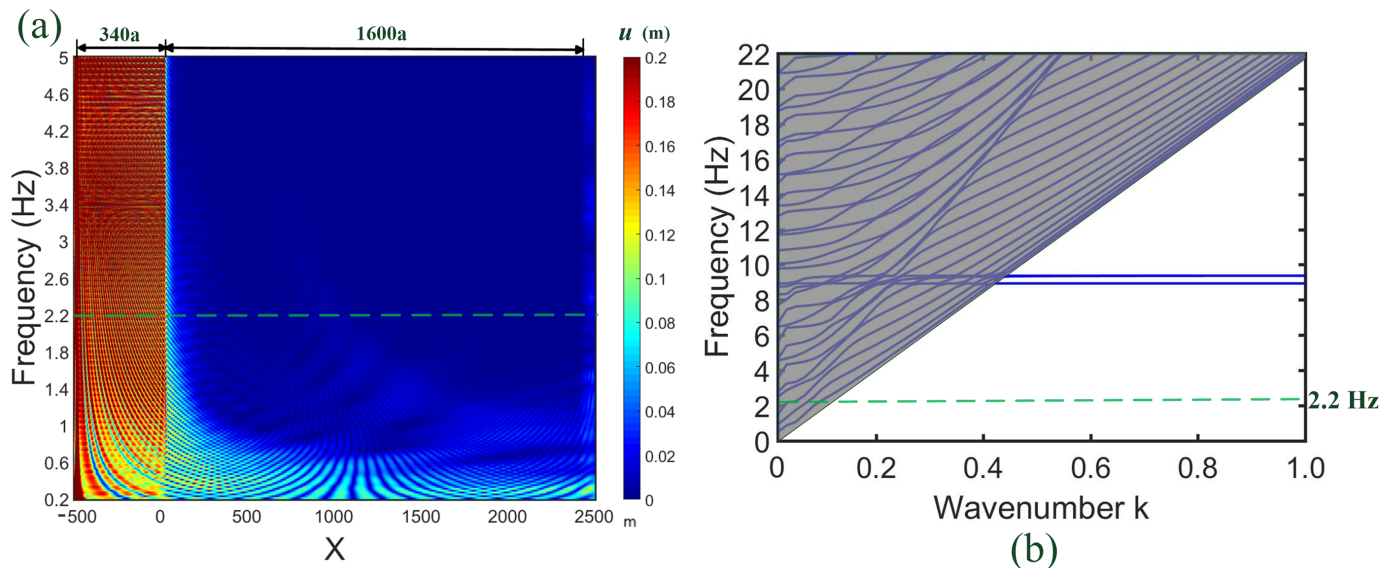

(b)
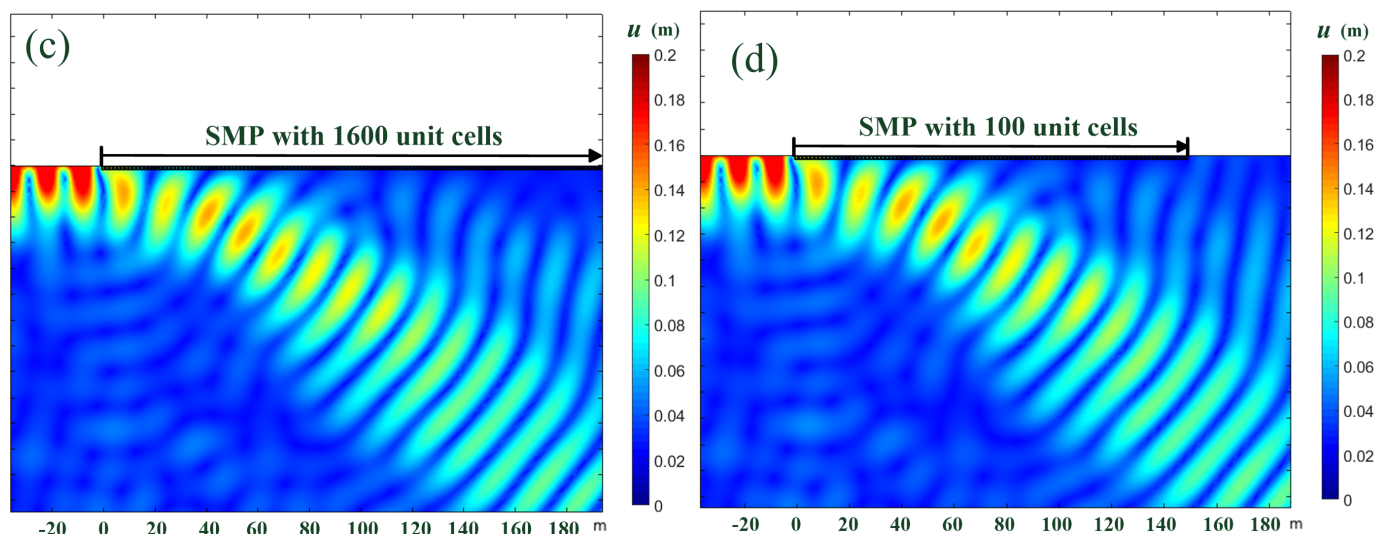

Figure4 

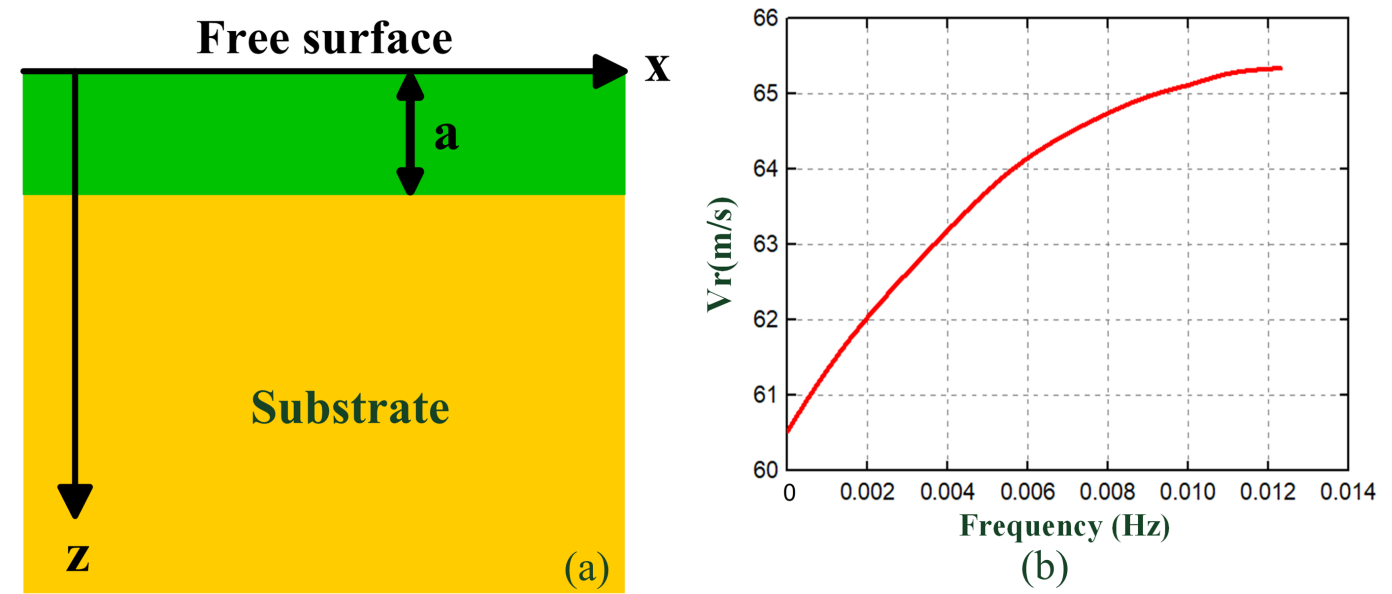

(b)

Figure5 

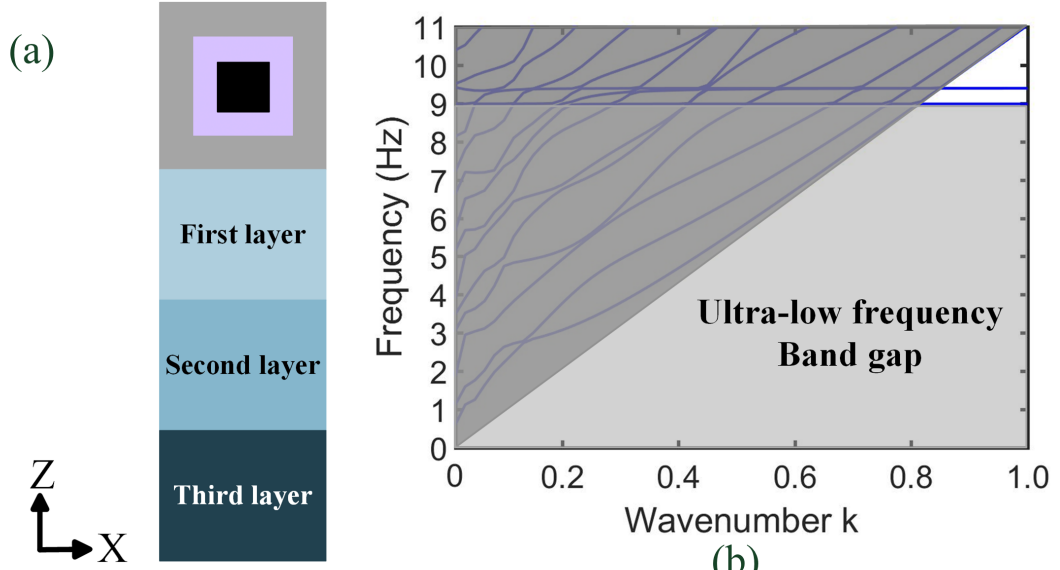

(b)

Figure6 

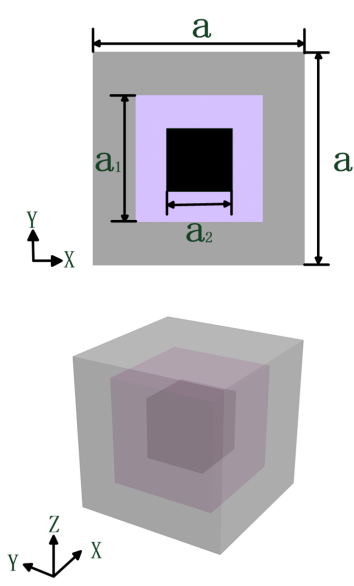

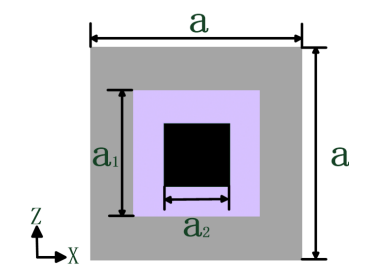

Figure7

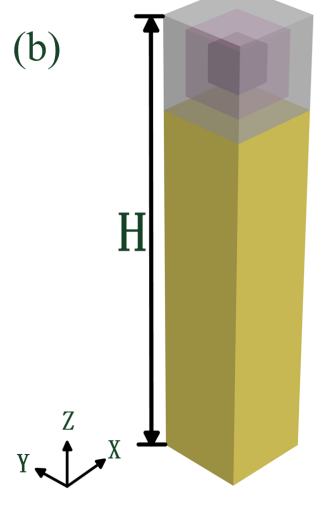



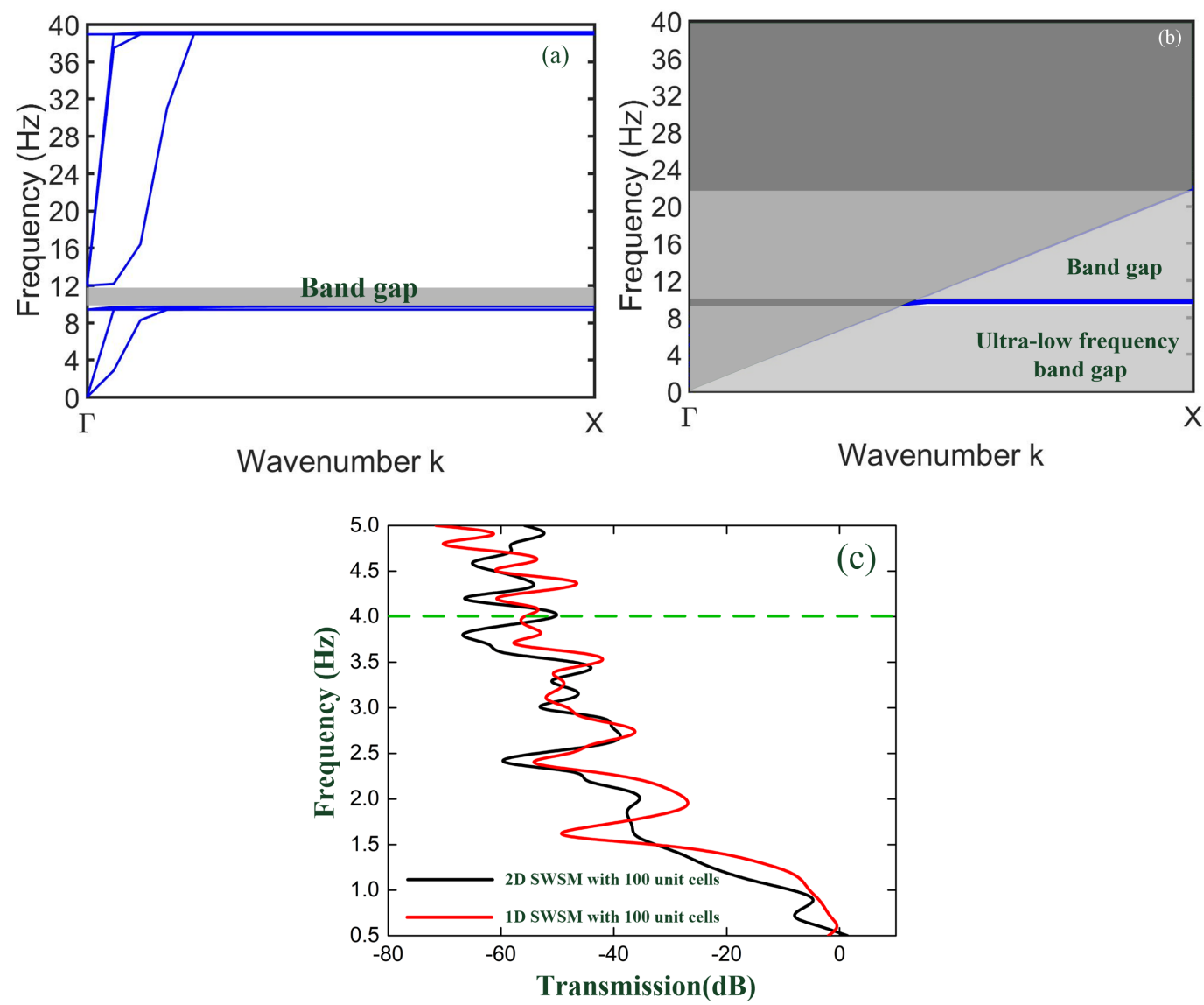

Figure8 


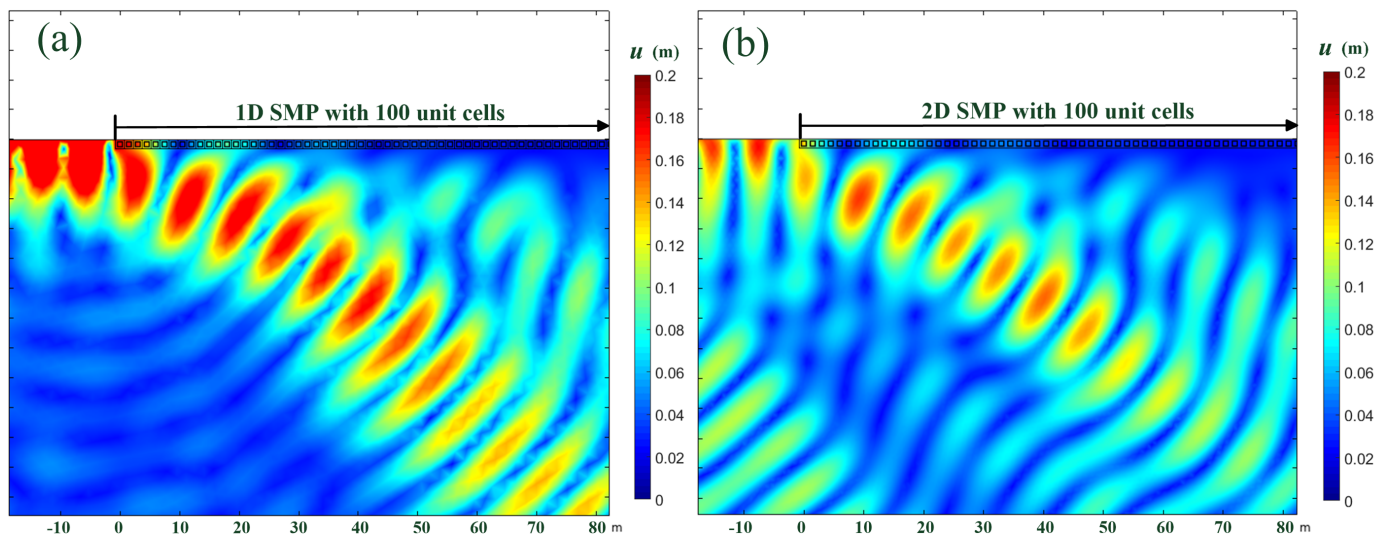

Figure9 
Table 1. Material parameters of the unit cell and the half space

\begin{tabular}{cccc}
\hline Material & Density $\left(\mathrm{kg} / \mathrm{m}^{3}\right)$ & Young's modulus $(\mathrm{Pa})$ & Poisson's ratio \\
\hline Rubber & 1300 & $1.2 \times 10^{5}$ & 0.47 \\
Concrete & 2500 & $4 \times 10^{10}$ & 0.3 \\
Steel & 7784 & $2.07 \times 10^{11}$ & 0.3 \\
foamed plate & 1053 & $1.6 \times 10^{5}$ & 0.39 \\
Soil & 1800 & $2 \times 10^{7}$ & 0.3 \\
\hline
\end{tabular}

